\title{
The Imaging Features of AIDS-Related Primary CNS Lymphoma; Case Series and Literature Review
}

\author{
Shunsuke Yanagisawa*, Yuya Sakakura, Shota Ozaki, Ryuichi Noda, Yuta Tamai, Makiko \\ Fujitani, Masato Inoue and Tetsuo Hara
}

Department of Neurosurgery, National Center for Global Health and Medicine, Tokyo, Japan

*Corresponding author: Shunsuke Yanagisawa, Department of Neurosurgery, National Center for Global Health and Medicine, 1-21-1, Toyama, Shinjuku-ku, Tokyo, Japan, Fax: +81-3-3207-1038, Tel: +81-3-3202-7181

\begin{abstract}
Primary CNS lymphoma is rare primary brain tumor. Meanwhile there are given number of acquired immune deficiency syndrome related primary CNS lymphoma. This brain tumor was reported that it showed ring enhancement in contrast MRI or CT. Here we analysed six acquired immune deficiency syndrome related primary CNS Iymphoma cases. All cases showed ring enhancement in contrast MRI. The central low uptake and peripheral high uptake were observed in 18F-fluorodeoxyglucose PET, and these imaging features may be useful to differ from other disease, especially toxoplasmosis. DWI in these six cases did not bring more diagnostic information. The numbers of tumor mass are single lesion of 5 cases and two lesions of one case. In histopathology, all six cases showed diffuse large B cell lymphoma, and five cases Epstein-Barr virus positive. Although the prognosis of this tumor was improved by introduction of combined antiretroviral therapy, standard therapy is not established.
\end{abstract}

\section{Abbreviations}

AIDS: Acquired Immune Deficiency Syndrome; AR-PCNSL: AIDS Related PCNSL; ART: Antiretroviral Therapy; DLBCL: Diffuse Large B Cell Lymphoma; FDG: 18F Fluorodeoxyglucose; PCNSL: Primary CNS Lymphoma

\section{Introduction}

Primary central nervous system lymphoma (PCNSL) accounts for $4.9 \%$ of primary brain tumor in Japan, $1.9 \%$ in USA $[1,2]$. PCNSL tends to develop in the immuno compromised patients [3]. Then there are given number of acquired immune deficiency syndrome (AIDS) related PCNSL (AR-PCNSL). Flinn reported that the incidence of AR-PCNSL was several thousand fold compared with
PCNSL of non AIDS patients [4]. Although there are many cases of human immunodeficiency virus (HIV) or AIDS in Europe and USA, those are few in Japan. Norden reported that AR-PCNSL accounted for $32.3 \%$ of all PCNSLs in Surveillance, Epidemiology, and End Results database [5]. However there are no precise reports from Japanese cohort due to few numbers of AIDS.

Then AR-PCNSL has several different clinical features compared with typical PCNSL. The typical PCNSL often shows uniform contrast enhancement in head $\mathrm{MRI}$ or $\mathrm{CT}$, and these imaging features were identified in $87 \%$ of PCNSL [6]. Uniform high uptake was also observed at PCNSL lesion in ${ }^{18} \mathrm{~F}$ fluorodeoxyglucose positron emission tomography (FDG-PET). However there are some reports that contrast MRI or CT showed ring like enhancement in AR-PCNSL $[7,8]$. Here we analysed the imaging features of six patients with AR-PCNSL and reviewed literatures.

\section{Case Series}

\section{Case 1}

A 42-year-old man presented headache and left hemiparesis of lower limb, and came to our office. We diagnosed his AIDS at 29-year-old and he had been given combined antiretroviral therapy (ART). Contrast MRI showed a ring enhancement lesion in right frontal lobe (Figure 1A). FDG-PET showed central low uptake and moderate uptake at tumor rim (Figure 1B). Meanwhile, diffusion weighted imaging (DWI) showed no clear findings (Figure 1C). At first, we suspected toxoplasmosis

Citation: Yanagisawa S, Sakakura Y, Ozaki S, Noda R, Tamai Y, et al. (2020) The Imaging Features of AIDS-Related Primary CNS Lymphoma; Case Series and Literature Review. Neurosurg Cases Rev 3:033. doi.org/10.23937/2643-4474/1710033

Accepted: April 29, 2020; Published: May 01, 2020

Copyright: (C) 2020 Yanagisawa S, et al. This is an open-access article distributed under the terms of the Creative Commons Attribution License, which permits unrestricted use, distribution, and reproduction in any medium, provided the original author and source are credited. 


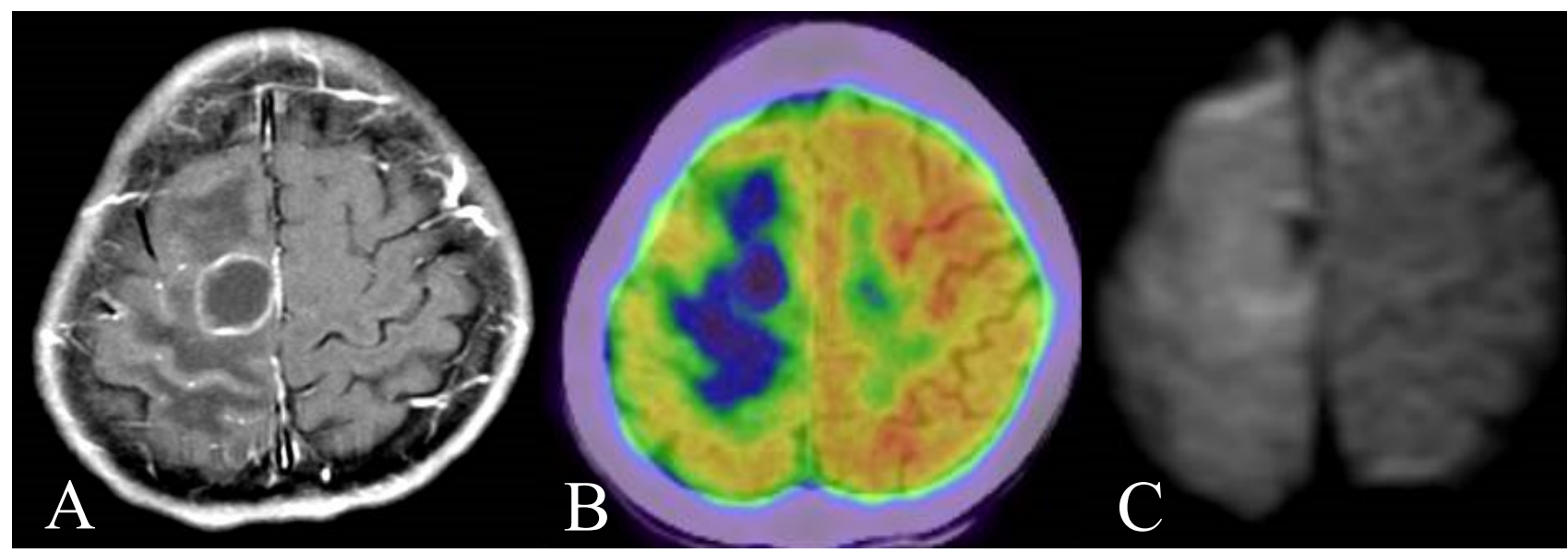

Figure 1: A) A 42-year-old man presented headache and left hemiparesis of lower limb. Contrast MRI showed a ring enhancement lesion in right frontal lobe; B) FDG-PET showed central low uptake and moderate uptake at tumor rim; C) DWI showed only edematous findings. Although we suspected toxoplasmosis at first, the histopathological findings were DLBCL.

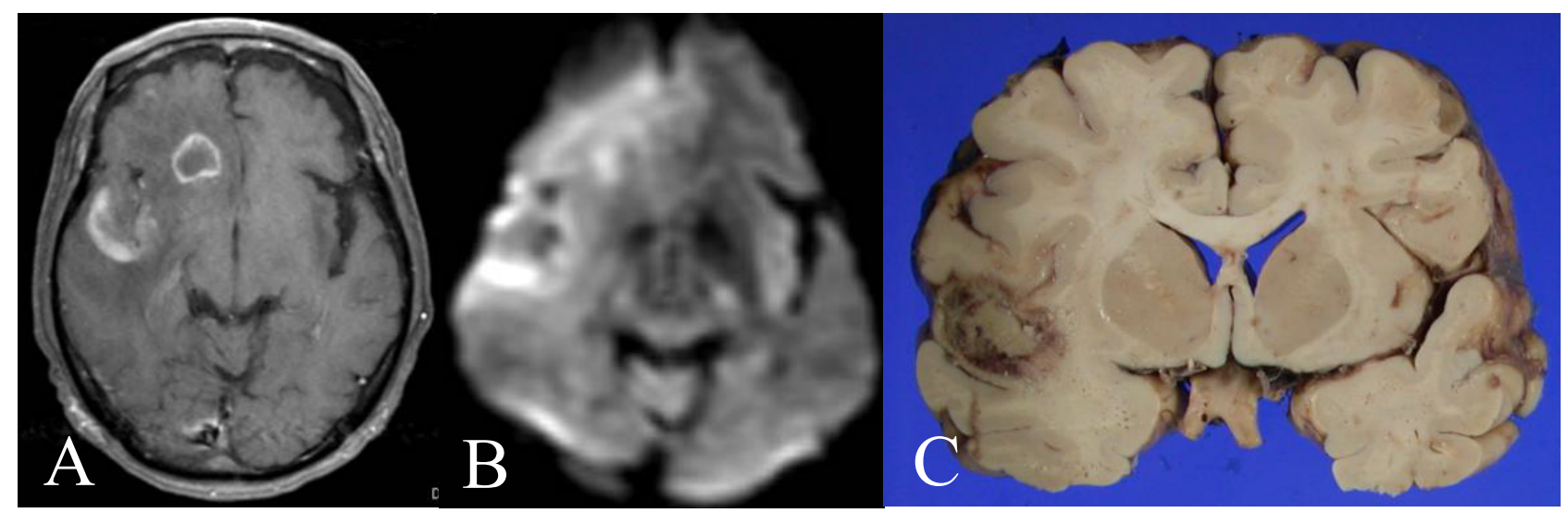

Figure 2: A 67 year-old man presented dysarthria and dysphagia. A) His head contrast MRI showed two ring enhancement lesions in both right frontal and temporal lobe; B) DWI showed no clear findings; C) His autopsy specimen showed central necrosis at the ring enhancement lesion of contrast MRI.

and performed stereotactic biopsy. The histopathological findings were diffuse large $B$ cell lymphoma (DLBCL) against our expectations. We performed whole brain radiotherapy as adjuvant therapy. Due to low CD4 positive $T$ cell in number, we could not induce chemotherapy. Although we expected his severe prognosis, he survives at 145 months.

\section{Case 2}

A 67-year-old man was indicated esophageal candidosis and we diagnosed his AIDS. Then he presented dysarthria and dysphagia at 7 months later. His head contrast MRI showed two ring enhancement lesions in both right frontal and right temporal lobe (Figure 2A). DWI showed no clear findings (Figure 2B). His histopathological diagnosis was DLBCL. He was high ages and low CD4 positive $T$ cell in number, and we performed whole brain radiotherapy alone to him. Then he died at 2 months from biopsy on the way of radiotherapy. We got his autopsy specimen and observed central necrosis at same lesion of ring enhancement (Figure 2C).

\section{Case 3}

37-year-old man presented refractory cutaneous ulcer and we diagnosed his AIDS. He presented dysarthria and gait disturbance 2 months later. His contrast MRI showed a ring enhancement at left caudate head (Figure 3A). FDG-PET showed central low uptake and moderate uptake at left putamen (Figure 3B). His histopathological specimen showed DLBCL (Figure $3 C$ ). His serum CD4 positive $T$ cell was normalized by combined $A R T$, and we performed rituximab plus high dose methotrexate therapy to him. He got complete remission and survives at 127 months after AR-PCNSL diagnosis.

\section{Case 4}

36-year-old man complained continuous dizziness and white moss inside the mouth. Three months later, his neighbor found that he was unconscious in his house and came to our office. Then we diagnosed his AIDS. His head MRI showed a ring enhancement at right temporal lobe (Figure 4A). FDG-PET showed central low uptake and peripheral moderate uptake (Figure 4B). His- 


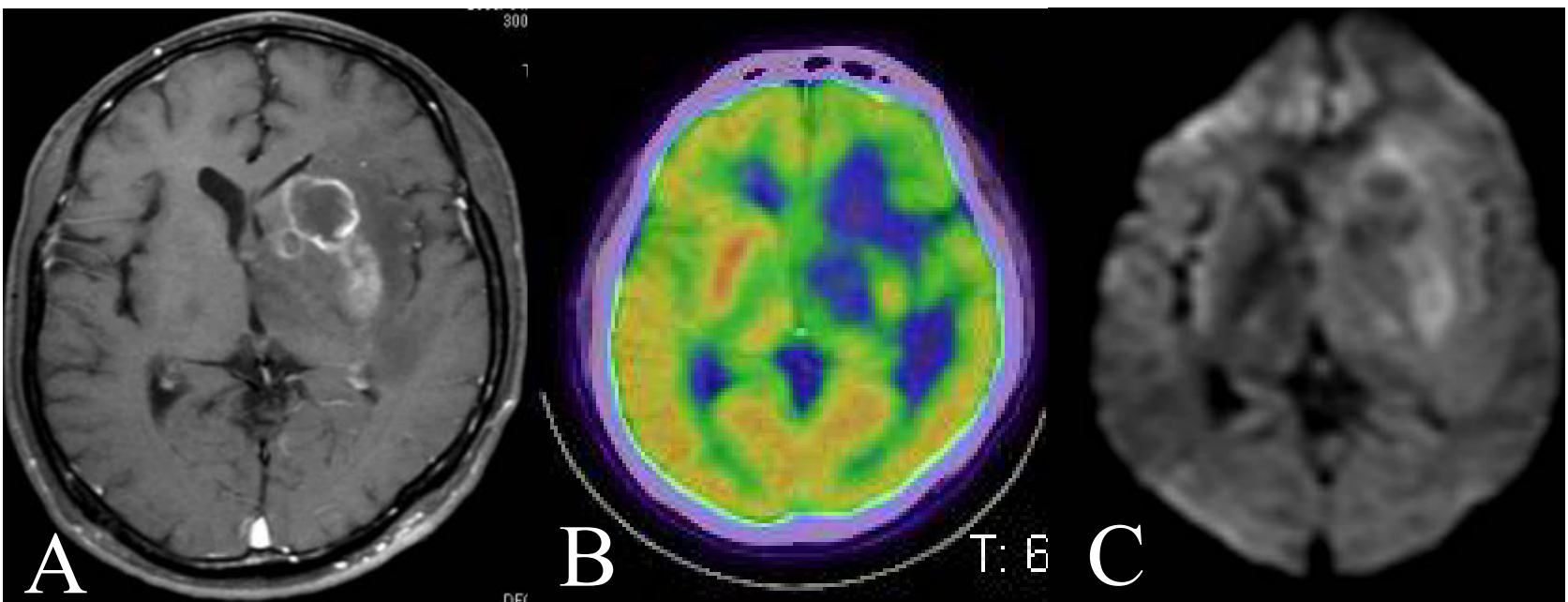

Figure 3: A 37 year-old man presented dysarthria and gait disturbance. A) His contrast MRI showed a ring enhancement at left caudate head; B) FDG-PET showed central low uptake and moderate uptake at left putamen; C) DWI showed slight high intensity at left putamen.

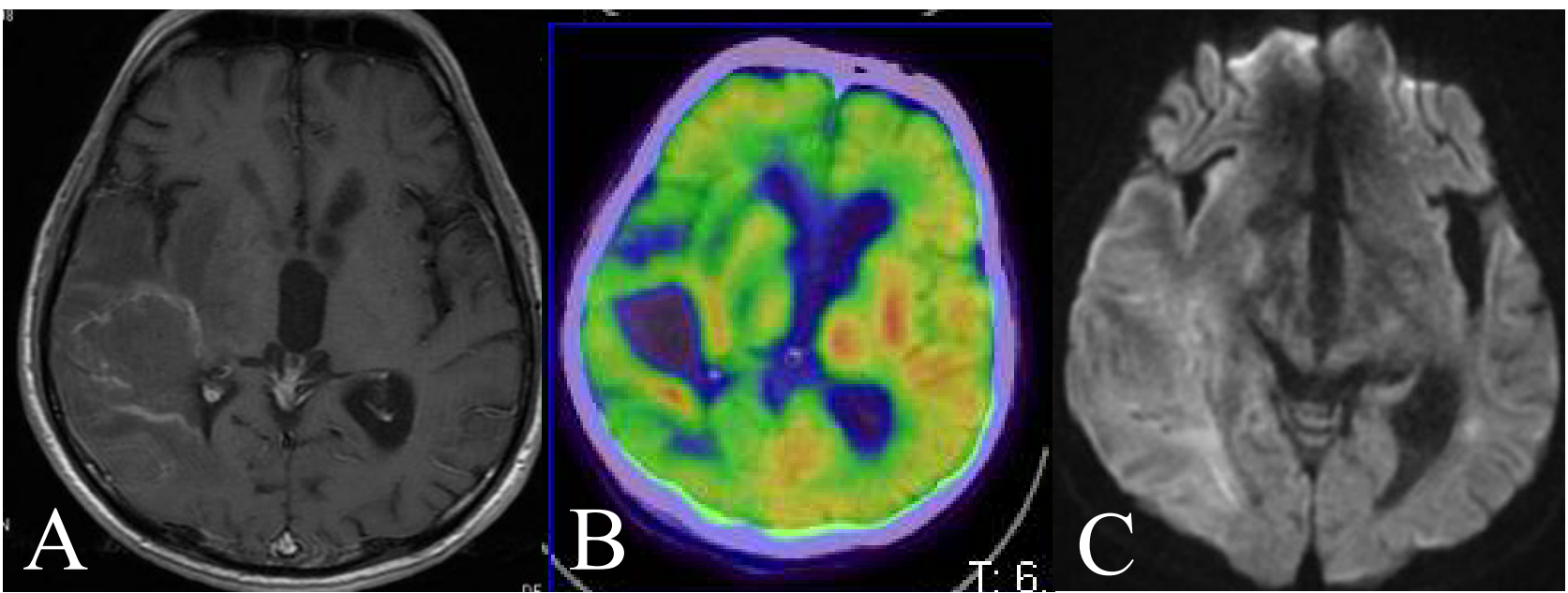

Figure 4: A 36 year-old man was unconscious in his house. A) His head MRI showed a ring enhancement at right temporal lobe; B) FDG-PET showed central low uptake and peripheral moderate or high uptake; C) DWI showed no clear findings.

topathological findings were DLCBL. Although we gave him combined ART and performed whole brain radiotherapy $50 \mathrm{~Gy} / 25$ fraction to him, he died at 6 months (Figure 4C).

\section{Case 5}

52-year-old man presented fever and dullness, and other doctor diagnosed his AIDS. The doctor introduced combined ART to him. His character changed 7 months later and came to our office. His head MRI showed a ring enhancement at right frontal lobe (Figure 5A). FDG-PET showed a ring like high uptake and central low uptake at same lesion (Figure 5B). Histopathological findings were DLBCL. Although we performed rituximab and radiotherapy to him, he died at 12 months (Figure 5C).

\section{Case 6}

28-year-old man presented dullness and we diagnosed his AIDS. His head MRI showed a ring enhancement at right caudate head (Figure 6A). FDG-PET showed ring like uptake and central low uptake at same lesion (Figure 6B). Histopathological findings were DL$\mathrm{BCL}$. His CD4 positive $\mathrm{T}$ cell in number improved by combined ART, we performed rituximab plus high dose methotrexate therapy for him (Figure 6C). He survives at 84 months.

All our six AR-PCNSL cases are summarized in Table 1.

\section{Discussion}

The central nervous system (CNS) disorder due to HIV and AIDS are as follow; 1) Opportunistic infection of immunodeficiency (60\%), 2) AR-PCNSL (a few \%), 3) Direct infection to CNS by HIV $(15 \%)[9,10]$. Opportunistic infection of immunodeficiency consists of toxoplasmosis, cryptococcosis, cytomegalovirus encephalitis and so on. Toxoplasmosis especially accounts for about half number of opportunistic infection of immunodeficiency. In Japanese cohort, Nishijima also reported that 10 cases of toxoplasmosis (17\%) and 3 cases of AR-PCNSL (5\%) were observed in 58 cases of AIDS patients [11].

In imaging, there are some brain tumors which show 


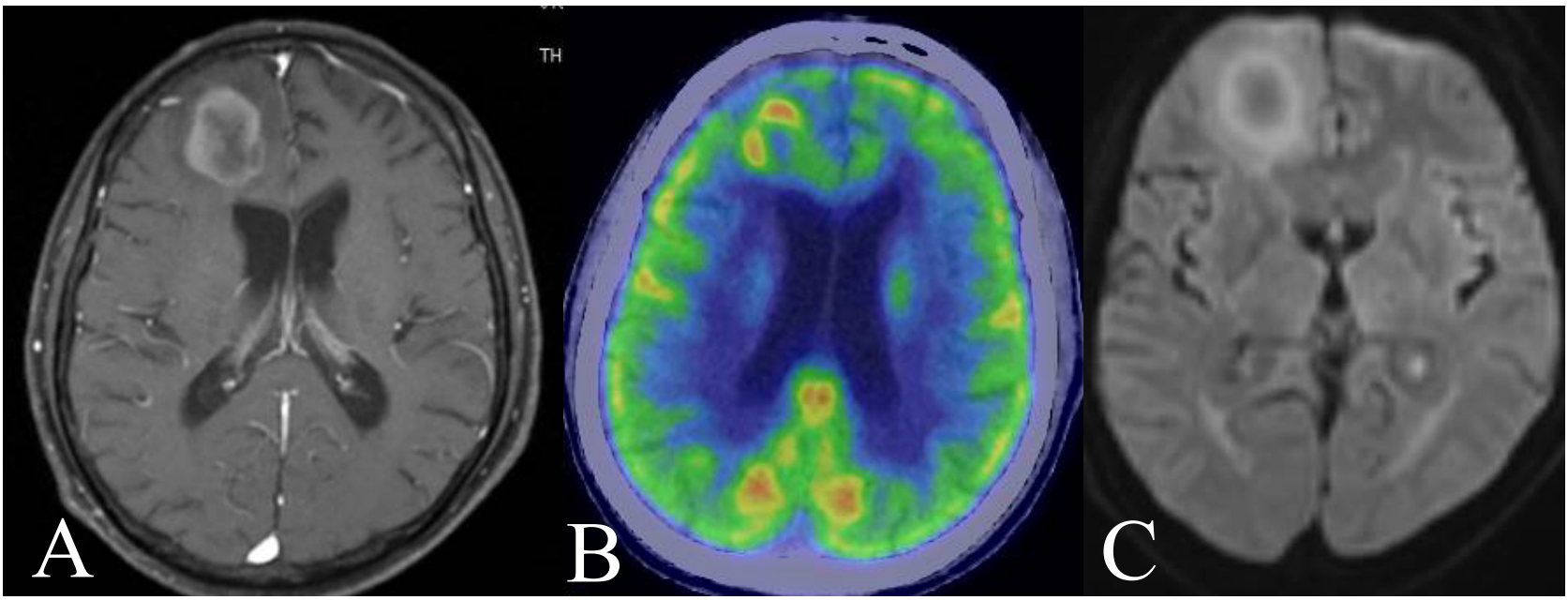

Figure 5: A 52 year-old man presented his character changed. A) His head MRI showed a ring enhancement at right frontal lobe; B) FDG-PET showed ring like high uptake and central low uptake at same lesion; C) DWI showed slight ring like high intensity.

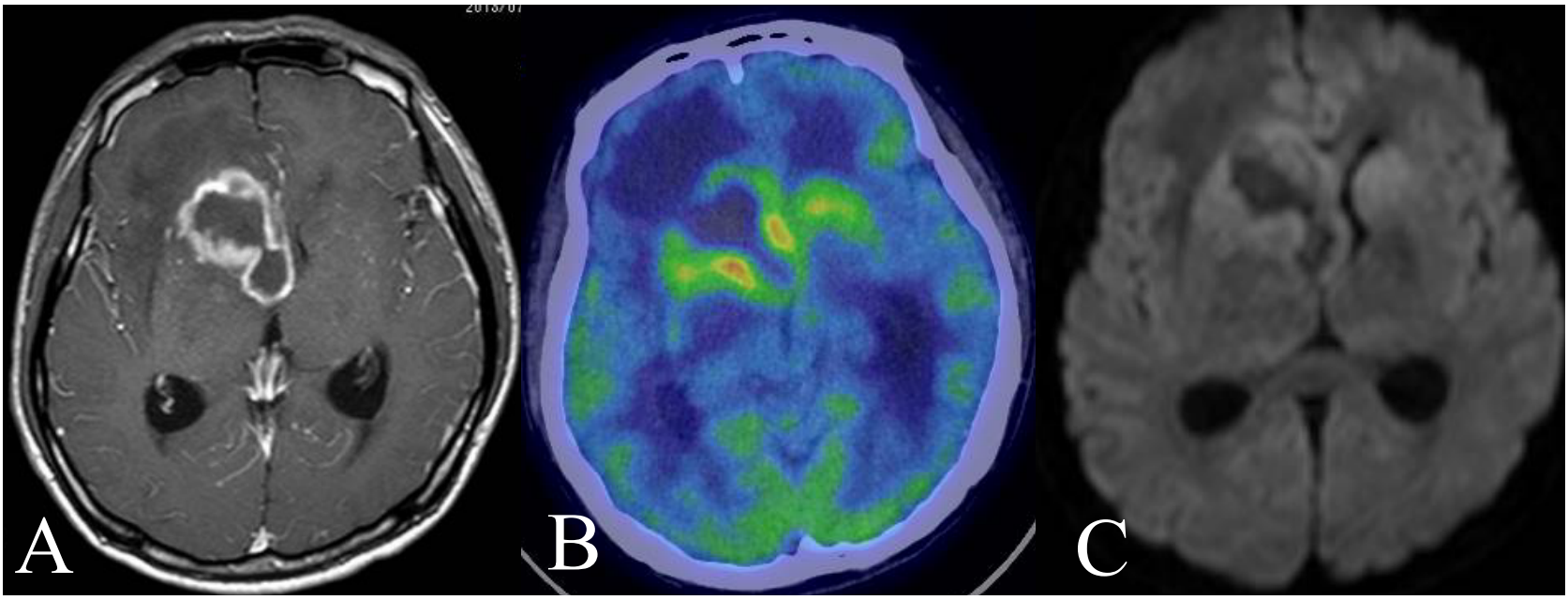

Figure 6: A 28 year-old man presented dullness. A) His head MRI showed a ring enhancement at right caudate head; B) FDG-PET showed ring like uptake and central low uptake at same lesion; C) DWI showed no clear findings.

ring like enhancement in contrast MRI or CT. They are high grade glioma, metastatic brain tumor, brain abscess and so on. However in patient with AIDS, toxoplasmosis is the first candidate, AR-PCNSL is second, and other diseases are very low frequency. Here the differential diagnosis between AR-PCNSL and toxoplasmosis is problem. As mentioned above, in frequency, it is natural that we suspect toxoplasmosis at first. In fact, we suspected that almost our cases would be toxoplasmosis at preoperative stage. It is difficult to diagnose the ring enhancement lesion in patients with AIDS by contrast MRI alone.

Although we analysed DWI as another sequence of MRI, DWI did not link to diagnosis of AR-PCNSL in our cases. Although DWI or apparent diffusion coefficient (ADC) map in MRI may be also effective, more researches are needed [12-14].

FDG-PET showed central low uptake and peripheral moderate or high uptake in our AR-PCNSL series, and this central low uptake reflects necrosis of tissue. In au- topsy (Case 2), necrosis was identified in center of low uptake, and these findings are compatible with imaging features. Westwood reported that FDG-PET showed no glucose metabolization in lesion of toxoplasmosis and it was effective in diagnosis [15]. The reason why ARPCNSL shows ring enhancement in contrast MRI is unclear. The necrosis may reflect tumor malignancy and aggressive progression.

The lesion numbers of our AR-PCNSL cases were single lesion in five cases and two lesions in one. Porter reported that in 21 toxoplasmosis cases, 18 cases (86\%) had multiple lesions [16]. Meanwhile Thurnher reported that AR-PCNSL showed 66 lesions in 22 patients [8]. Both toxoplasmosis and AR-PCNSL are generally multiple lesions unlike our research.

The purpose of surgery in PCNSL is not tumor mass reduction but diagnosis by biopsy. The imaging feature of typical PCNSL is very specific, and it is easy to make preoperative surgical plan. In biopsy of AR-PCNSL, both contrast MRI and FDG-PET are useful as well as typical 


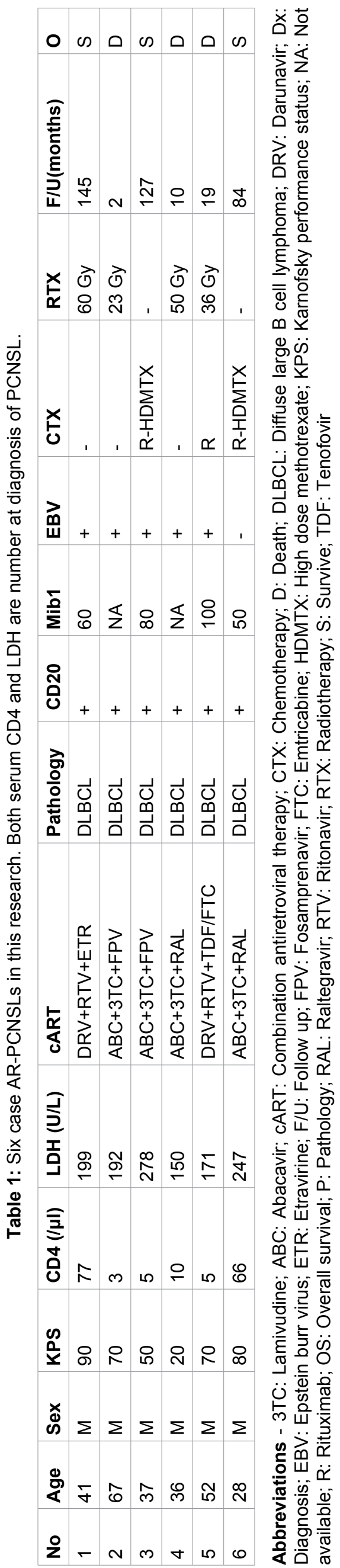

PCNSL. The ideal target of biopsy is the high uptake part of FDG-PET.

In pathological findings, our case series were all DL$\mathrm{BCL}(100 \%)$ and Haldorsen reports same result (100\%) [17]. DeAngelis reported that 11 cases of 13 AR-PCNSLs $(85 \%)$ were Epstein-Barr virus positive, and this result came close to ours (83\%) [18].

In symptom, focal signs of CNS were observed in our six cases and diagnostic clues. Although no symptomatic seizures were observed in our case series, there were two reports that the patients with AR-PCNSL had more symptomatic seizures compared with non AR-PCNSL $[19,20]$. Meanwhile, Holtzman reported that symptomatic seizure were observed in $15-30 \%$ of patients with toxoplasmosis or AR-PCNSL, and it was difficult to diagnose these disease in view point of symptomatic seizure [21].

There are no standard therapy for AR-PCNSL. In 1980 s when there were no ART, Baumgartner reported that 55 cases of AR-PCNSL were performed whole brain radiotherapy $40 \mathrm{~Gy}$, and their median overall survival time was only 3.9 months [22]. After introduction of ART, 5 year survival rate improved to $22.8 \%$ [23]. In Japanese cohort, Nagai reported that 3 year survival rate was $64 \%$ by performing whole brain radiotherapy $30 \mathrm{~Gy}$ for AR-PCNSL [24]. Patients with AIDS had low CD4 positive $T$ cell in number, and it is difficult for them to introduce chemotherapy which brings about pancytopenia. Jacomet reported that although methotrexate, thiotepa and procarbazine were performed for AR-PCNSL, median overall survival time was only 9 months [25]. Shah reported that high dose methotrexate therapy was performed for AR-PCSNL treated by ART and median overall survival time was 19 months [26]. By combined ART, increasing chance of performing chemotherapy contributes to improve the prognosis of AR-PCNSL. More researches need to establish the standard therapy for AR -PCNSL.

\section{Conclusion}

Head contrast MRI in patient with AR-PCNSL showed ring enhancement unlike typical PCNSL. It is important to differ AR-PCSNL from toxoplasmosis, and FDG-PET may be effective modality in preoperative diagnosis.

\section{Conflict of Interest}

All authors have no declaration for conflict of interest associated with this research.

\section{Acknowledgement}

We are thankful for collecting data to Dr. Oka and Dr. Kikuchi who belong to AIDS Clinical Center of National Center for Global Health and Medicine.

\section{Funding}

No funding was received for this study. 


\section{Ethical Approval}

All procedures performed in the studies involving human participants were in accordance with the national research committee and with the 1964 Helsinki Declaration and its later amendments or comparable ethical standards.

\section{Informed Consent}

Informed consent was obtained from all individual participants included in the study.

\section{Contributions}

\section{Conception and design: Yanagisawa.}

Acquisition of data: Yanagisawa, Sakakura, Ozaki, Noda, Tamai, Fujitani, Inoue.

Analysis and interpretation of data: Yanagisawa.

Drafting the article or revisiting it critically of important intellectual content: Hara.

\section{References}

1. The Committee of Brain Tumor Registry of Japan (2017) Report of Brain tumor registry of japan (2005-2008). Neurol Med Chir (Tokyo) 57: 9-102.

2. Ostrom QT, Cioffi G, Gittleman H, Patil N, Waite K, et al. (2019) CBTRUS statistical report: Primary brain and other central nervous system tumors diagnosed in the United States in 2012-2016. Neuro Oncol 21.

3. Gibson TM, Engels EA, Clarke CA, Lynch CF, Weisenburger DD, et al. (2014) Risk of diffuse large B-cell lymphoma after solid organ transplantation in the United States. Am J Hematol 89: 714-720.

4. Flinn IW, Ambinder RF (1996) AIDS primary central nervous system lymphoma. Curr Opin Oncol 8: 373-376.

5. Norden AD, Drappatz J, Wen PY, Claus EB (2011) Survival among patients with primary central nervous system lymphoma, 1973-2004. J Neurooncol 101: 487-493.

6. Kuker W, Nagele T, Korfel A, Heckl S, Thiel E, et al. (2005) Primary central nervous system lymphomas (PCNSL): MRI features at presentation in 100 patients. J Neurooncol 72: 169-177.

7. Chang L, Cornford ME, Chiang FL, Ernst TM, Sun NC, et al. (1995) Radiologic-pathologic correlation. Cerebral toxoplasmosis and lymphoma in AIDS. AJNR Am J Neuroradiol 16: 1653-1663.

8. Thurnher MM, Rieger A, Kleibl-Popov C, Settinek U, Henk C, et al. (2001) Primary central nervous system lymphoma in AIDS: A wider spectrum of CT and MRI findings. Neuroradiology 43: 29-35.

9. Chimelli L, Rosemberg S, Hahn MD, Lopes MB, Netto MB (1992) Pathology of the central nervous system in patients infected with the human immunodeficiency virus (HIV): A report of 252 autopsy cases from Brazil. Neuropathol Appl Neurobiol 18: 478-488.

10. Matthiessen L, Marche C, Labrousse F, Trophilme D, Fontaine C, et al. (1992) Neuropathology of the brain in 174 patients who died of AIDS in a Paris hospital 1982-1988. Ann Med Interne 143: 43-49.

11. Nishijima T, Gatanaga H, Teruya T, Tajima T, Kikuchi $Y$, et al. (2014) Brain magnetic resonance imaging screening is not useful for HIV-1-infected patients without neurological symptoms. AIDS Res Hum Retroviruses 30: 970-974.
12. Camacho DL, Smith JK, Castillo M (2003) Differentiation of toxoplasmosis and lymphoma in AIDS patients by using apparent diffusion coefficients. AJNR Am J Neuroradiol 24: 633-637.

13. Schroeder PC, Post MJ, Oschatz E, Stadler A, Bruce-Gregorios J, et al. (2006) Analysis of the utility of diffusion-weighted MRI and apparent diffusion coefficient values in distinguishing central nervous system toxoplasmosis from lymphoma. Neuroradiology 48: 715-720.

14. Haldorsen IS, Krakenes J, Krossnes BK, Mella O, Espeland A (2009) CT and MR imaging features of primary central nervous system lymphoma in Norway, 1989-2003. AJNR Am J Neuroradiol 30: 744-751.

15. Westwood TD, Hogan C, Julyan PJ, Coutts G, Bonington S, et al. (2013) Utility of FDG-PETCT and magnetic resonance spectroscopy in differentiating between cerebral lymphoma and non-malignant CNS lesions in HIV-infected patients. Eur J Radiol 82: e374-e379.

16. Porter SB, Sande MA (1992) Toxoplasmosis of the central nervous system in the acquired immunodeficiency syndrome. N Engl J Med 327: 1643-1648.

17. Haldorsen IS, Krakenes J, Goplen AK, Dunlop O, Mella O, et al. (2008) AIDS-related primary central nervous system lymphoma: A Norwegian national survey 1989-2003. BMC Cancer 8: 225.

18. DeAngelis LM, Wong E, Rosenblum M, Furneaux H (1992) Epstein-Barr virus in acquired immune deficiency syndrome (AIDS) and non-AIDS primary central nervous system lymphoma. Cancer 70: 1607-1611.

19. Fine HA, Mayer RJ (1993) Primary central nervous system lymphoma. Ann Intern Med 119: 1093-1104.

20. Maher EA, Fine HA (1999) Primary CNS lymphoma. Semin Oncol 26: 346-356.

21. Holtzman DM, Kaku DA, So YT (1989) Newonset seizures associated with human immunodeficiency virus infection: Causation and clinical features in 100 cases. Am J Med 87: 173-177.

22. Baumgartner JE, Rachlin JR, Bckstead JH, Meeker TC, Levy RM, et al. (1990) Primary central nervous system lymphomas: Natural history and response to radiation therapy in 55 patients with acquired immunodeficiency syndrome. $\mathrm{J}$ Neurosurg 73: 206-211.

23. Gopal S, Patel MR, Yanik EL, Cole SR, Achenbach CJ, et al. (2013) Temporal trends in presentation and survival for HIV-associated lymphoma in the antiretroviral therapy era. J Natl Cancer Inst 105: 1221-1229.

24. Nagai $H$, Odawara T, Ajisawa A, Tanuma J, Hagiwara S, et al. (2010) Whole brain radiation alone produces favourable outcomes for AIDS-related primary central nervous system lymphoma in the HAART era. Eur J Haematol 84: 499-505.

25. Jacomet C, Girard PM, Lebrette MG, Farese VL, Monfort L, et al. (1997) Intravenous methotrexate for primary central nervous system non-Hodgkin's lymphoma in AIDS. AIDS 11: $1725-1730$.

26. Shah GD, Yahalom J, Correa DD, Lai RK, Raizer JJ, et al. (2007) Combined immunochemotherapy with reduced whole-brain radiotherapy for newly diagnosed primary CNS lymphoma. J Clin Oncol 25: 4730-4735.

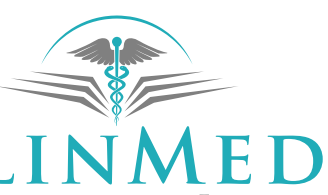

INTERNATIONAL LIBRARY 